


\section{Frohe Weihnachten}

\section{$06 / 10$}

\section{Inhalt}

\section{brief des herausgebers}

\section{8 „pädiatrie \& pädologie“: Jahresrückblick 2010} und -ausblick 2011

Univ.-Prof. Dr. Reinhold Kerbl, Vorstand der Abteilung für Kinder- und Jugendheilkunde am Landeskrankenhaus Leoben und Herausgeber von „pädiatrie \& pädologie“"

\section{allergologie}

\section{Allergen-spezifische Immuntherapie bei Kindern und} Jugendlichen: Fakten und häufig gestellte Fragen Zsolt Szépfalusi, MD, Saskia Gruber, MD, Eiwegger Thomas, MD, Eleonora Dehlink, MD, PhD; Univ.-Klinik für Kinder- und Jugendheilkunde der Medizinischen Universität Wien

\section{serie stillen}

22 Zufütterung bei gesunden, gestillten Neugeborenen Dr. Alfred Dilch, G. v. Preyer'sches Kinderspital, Wien DKKS Doris Teufel IBCLC, LKM Scheibbs

\section{chirurgie}

\section{Die Biegungsfraktur des Unterarmes im Kindesalter}

Dr. Simon Kargl, Dr. Jörg Breitwieser, Univ.-Doz. Prim. Dr. Wolfgang Pumberger; Abteilung für Kinder- und Jugendchirurgie, Landes-Frauenund Kinderklinik Linz

\section{gesundheitswissenschaften}

\section{Die Balanced Score Card in der Pädiatrie}

Dr. med. Dipl. oec. med. Jürgen Brunner, geschäftsführender Oberarzt am Department für Kinder- und Jugendheilkunde der Medizinischen Universität Innsbruck

\section{reisebericht}

\section{Besuch in Ghana, Westafrika}

Em. Univ.-Prof. Dr. Ronald Kurz, Graz

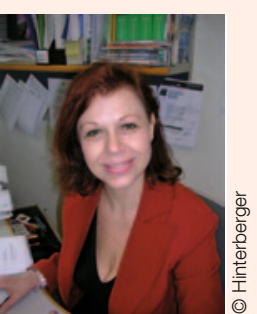

Wieder einmal ist es soweit: Wir überreichen Ihnen unser Heft 6 , das letzte aus 2010!

Univ.-Prof. Dr. Reinhold Kerbl präsentiert seinen zur lieben Tradition gewordenen "Brief des Herausgebers".

Auch zur Tradition ist es geworden, dass „pädiatrie \& pädologie“ bei den beiden hochkarätigen pädiatrischen Tagungen, welche alljährlich im Jänner unter der Leitung von Univ.-Prof. Dr. Karl Zwiauer und Univ.-Prof. Dr. Wolfgang Sperl stattfinden, aufliegt. Wir freuen uns, auf diese Weise unsere treuen Leser im verschneiten Obergurgl zu entspanntem Lesegenuss verführen und selbstverständlich auch Brücken zu neuen Interessenten bauen zu dürfen.

In unserer Dezemberausgabe erwartet Sie eine umfassende, bunte Palette an Beiträgen. Besonders hervorheben möchten wir zwei Beiträge, die nicht alltäglich sind: Em. Univ.-Prof. Dr. Ronald Kurz hat Ghana besucht - seinen spannenden Reisebericht finden Sie in dieser Ausgabe. Dr. Jürgen Brunner befasst sich neben der Pädiatrie auch mit Gesundheitswissenschaften; er berichtet über die Balanced Score Card und ihre Anwendung in der Pädiatrie.

Das Jahr 2010 war für die meisten von uns ereignisreich und barg so manche Klippe. Was uns, Herausgeber, Redaktion, Herstellung und Verkauf der „pädiatrie \& pädologie" betrifft, so können wir stolz sein: Die Mühe hat sich gelohnt! Sechs Ausgaben, die sich sehen lassen, wurden publiziert. Danke, liebe Leserinnen und Leser, für Ihr geschätztes Interesse und Ihre Treue!

Frohe Weihnachten und alles Gute für 2011!

Dr. Renate Höhl 